\title{
ASPERGILOSIS CEREBRAL: REPORTE DE CASO
}

\author{
(Brain aspergillosis: a case report)
}

Eduardo Contreras Zúñiga (1)

Gloria Elena Posadan (2)

Sandra Ximena Zuluaga Martínez (3)

(1) Medicina Interna Universidad del Valle.

Cali. Colombia. Correspondencia: edo11@hotmail.com.

Dirección: Calle 4 No, 65-14. Cali. Colombia.

(2) Medicina Interna. Clínica de Occidente S.A. Cali. Colombia.

(3) Cirujano general. Angiografía se Occidente SA.

Cali. Colombia.

Palabras claves: Aspergilosis cerebral, aspergilosis invasiva.

Keywords: Brain aspergillosis, invasive aspergillosis.

\section{RESUMEN}

En los últimos años la incidencia por infecciones de hongos del genero Aspergillus han aumentado de forma significativa como consecuencia del incremento de pacientes con estados de inmunodepresión, fundamentalmente por la alta incidencia de SIDA y por el mayor empleo de tratamientos inmunosupresores. Las aspergilosis son infecciones infrecuentes en el sistema nervioso central, constituyendo el $4 \%$ de las enfermedades invasoras, tratandose de una entidad grave, con un pobre pronóstico (mortalidad mayor del 95\%). Se presenta un caso clínico de un paciente masculino de 48 años, con miocardiopatia dilatada terminal que fue sometido a transplante cardiaco, tratamiento inmunosupresor y profilaxis antimicrobiana. Después de su alta ( 23 días post cirugia), regresa con compromiso del sensorio y focalidad neurológica, por lo que se practica RNM que evidenció lesión microangiopatica, con edema y realce periférico anular. Cultivos microbiológicos negativos y LCR dentro de lo normal. Posteriormente (10 días después del ingreso) empeora su condición neurológica e imagenológica, por lo que se realizó biopsia estereotaxica obteniéndose exámenes directos negativos e histología sin evidencia de microorganismos, sin embargo, en los cultivos se obtuvo Aspergillus fumigatus. Se inició tratamiento con Voriconazol con buena respuesta inicial, pero posteriormente empeora su condición neurológica y general, con signos y síntomas de rechazo del transplante cardiaco, falleciendo a los 62 días postransplante.

Recibido el 18 de Diciembre 2008

Aceptado el 30 de Diciembre 2008

\section{SUMMARY}

In recent years the incidence of fungal infections of the genera Aspergillus have increased significantly as a result of the increase in patients with immunocompromised states, primarily by the high incidence of AIDS and the increased use of immunosuppressive treatments. The aspergillosis infections are rare in the central nervous system, constituting $4 \%$ of invasive disease, in the case of a serious entity with a poor prognosis (mortality greater than 95\%). We report a case of a 48-year-old male patient with terminal dilated cardiomyopathy underwent heart transplantation, immunosuppressive therapy and antimicrobial prophylaxis. After his high (23 days post-surgery), is back with commitment and focal neurologic consciousness, so that is practiced MRI showed microangiopathic injury, edema and peripheral enhancement cancel. Microbiological cultures and negative CSF within normal. Later (10 days after admission) her neurological condition worsened and imagery, so stereotactic biopsy was obtained and histology tests negative without direct evidence of microorganisms, however the culture was positive to Aspergillus fumigatus. Voriconazole treatment started with good initial response, but later his condition worsened neurological and general signs and symptoms of heart transplant rejection, died 62 days postranplantation.

\section{INTRODUCCION}

En la última década se ha producido un incremento sustancial de los casos de aspergilosis invasiva como consecuencia de la generalización de tratamientos 
inmunosupresores cada vez más potentes. Este incremento en la frecuencia de esta enfermedad no se ha visto acompañado de una reducción en la mortalidad, que sigue siendo elevada $(1,2)$.

La aspergilosis pulmonar es, sin duda, la forma clínica más frecuente de presentación de esta entidad, seguida por la sinusitis, la infección del árbol traqueobronquial y la del sistema nervioso central $(2,10)$.

La aspergilosis cerebral puede verse en un $4 \%$ hasta un $20 \%$ de las formas invasivas. El diagnóstico precoz de la infección por Aspergillus del Sistema Nervioso Central (SNC) es un gran desafío, donde la sospecha clínica fundamentada es clave por la alta letalidad de la entidad aún con tratamiento oportuno. La importante dificultad diagnóstica es favorecida por algunas características de la entidad: baja incidencia, compromiso de individuos inmunocomprometidos que presentan múltiples infecciones, manifestaciones neurológicas clínicas e imagenológicas inespecíficas y métodos diagnósticos etiológicos de resultados tardíos $(1,3,4)$.

\section{CASO CLINICO}

Paciente masculino, de 48 años de edad, con antecedentes de miocardiopatía dilatada idiopática, por la que fue sometido a transplante cardíaco, recibiendo dentro del protocolo de manejo perioperatorio profilaxis antibiótica con Ceftriaxona y Vancomicina, además de bolos de Metilprednisolona. Se adicionó Micofenolato Mofetil y Ciclosporina. También se inicio profilaxis con Trimetropinsulfametoxazol (TMS), Aciclovir, Nistatina y Pirimetamina (seronegativo para anticuerpos contra Toxoplasma gondii). Se realizaron ecocardiogramas transtorácicos que evidenciaban adecuado funcionamiento del injerto. Se realizaron 2 biopsias endomiocárdicas (Semana 1 y 2) negativas para rechazo celular del injerto. El paciente es dado de alta a los 18 días después del transplante para seguir con Ciclosporina, Prednisolona, Micofenolato Mofetil, Aciclovir, Nistatina, ASA, Omeprazol, Calcio, Calcitriol, Enalapril, Metoprolol, TMS.

El paciente reingresa a los 23 días postransplante por 72 horas de evolución de malestar general, disnea subjetiva e irritabilidad. Al examen físico se encontró en regular estado general, TA 126/72 mmHg, Fc:100 l.p.m., Fr:18, sin hallazgos anormales al examen físico. Paraclínicos evidencian sodio: $118 \mathrm{mEq} / \mathrm{lt}$, potasio: $5.2 \mathrm{mEq} / \mathrm{lt}$. Resto de los estudios dentro de límites normales. Ecocardiograma evidencia hipertrofia marcada del ventrículo izquierdo con fracción de eyección del 65\% 24 horas después del ingreso:

A pesar de la corrección de las alteraciones hidroelectrolíticas, se observa mayor deterioro neurológico, confusión y desorientación.

A los 25 días postrasplante: Se observa mutista, no obe- dece ordenes, hay respuesta plantar flexora bilateral y marcado temblor distal en miembros superiores. Se realiza una resonancia magnética cerebral (RMN) (Fig. 1, 2, 3) observándose múltiples lesiones secundarias a microangiopatía con edema perilesional y realce periférico anular. Se solicita una tomografía de tórax sin lesiones específicas. Los hemocultivos fueron negativos y Galactomannano fue negativo en dos oportunidades. EL fisicoquímico y citológico del LCR estaba dentro de los parámetros normales, con cultivo negativo.

El paciente evoluciona en forma favorable durante los siguientes 7 días.

A los 32 días postrasplante: Control escanográfico (Fig. 4 y 5) evidencia mejoría parcial de las lesiones. El paciente permaneció con temblor distal pero con mejoría neurológica importante.

A los 37 días postrasplante: Presenta episodio convulsivo, relajación de esfínteres, sialorrea y desviación de la mirada. Se realiza nueva resonancia magnética cerebral que muestra aumento del edema perilesional, aparición de nuevas lesiones con efecto de masa y realce periférico (Fig.6 y 7).

A los 40 días postrasplante: Se realiza biopsia cerebral estereotáxica obteniendo abundante material purulento. El examen directo del material coloreado con Gram fue negativo; en el estudio histológico se observo necrosis sin evidencia de microorganismos.

A los 44 días postrasplante: El paciente se deteriora neurológicamente, presenta hemiparesia. El reporte preliminar del cultivo es informado como positivo para un hongo filamentoso el cual, posteriormente fue tipificado como un Aspergillus fumigatus. Se inicia tratamiento con Voriconazol $4 \mathrm{mg} / \mathrm{kg}$ cada 12 horas.

A los 50 días postrasplante: El paciente mejora neurológicamente, queda secuela de hemiparesia izquierda. Se realiza control escanográfico que evidencia mejoría de todas las lesiones previas excepto una a nivel de de hemisferio cerebral izquierdo.

A los 55 días postrasplante: El paciente presenta disnea progresiva, cifras tensionales bajas y disminución de fracción de eyección (30\%) mediante ecocardiograma. Teniendo en cuenta la mejoría neurológica y radiológica del paciente, se reajusta dosis de esteroide para manejo del rechazo celular con compromiso Hemodinámico.

A los 60 días postrasplante: Empeoramiento de hemiparesia izquierda y disartria. Se realiza nuevo estudio imagenólogico (Fig. 8 y 9) que evidencia sangrado intraparen- 

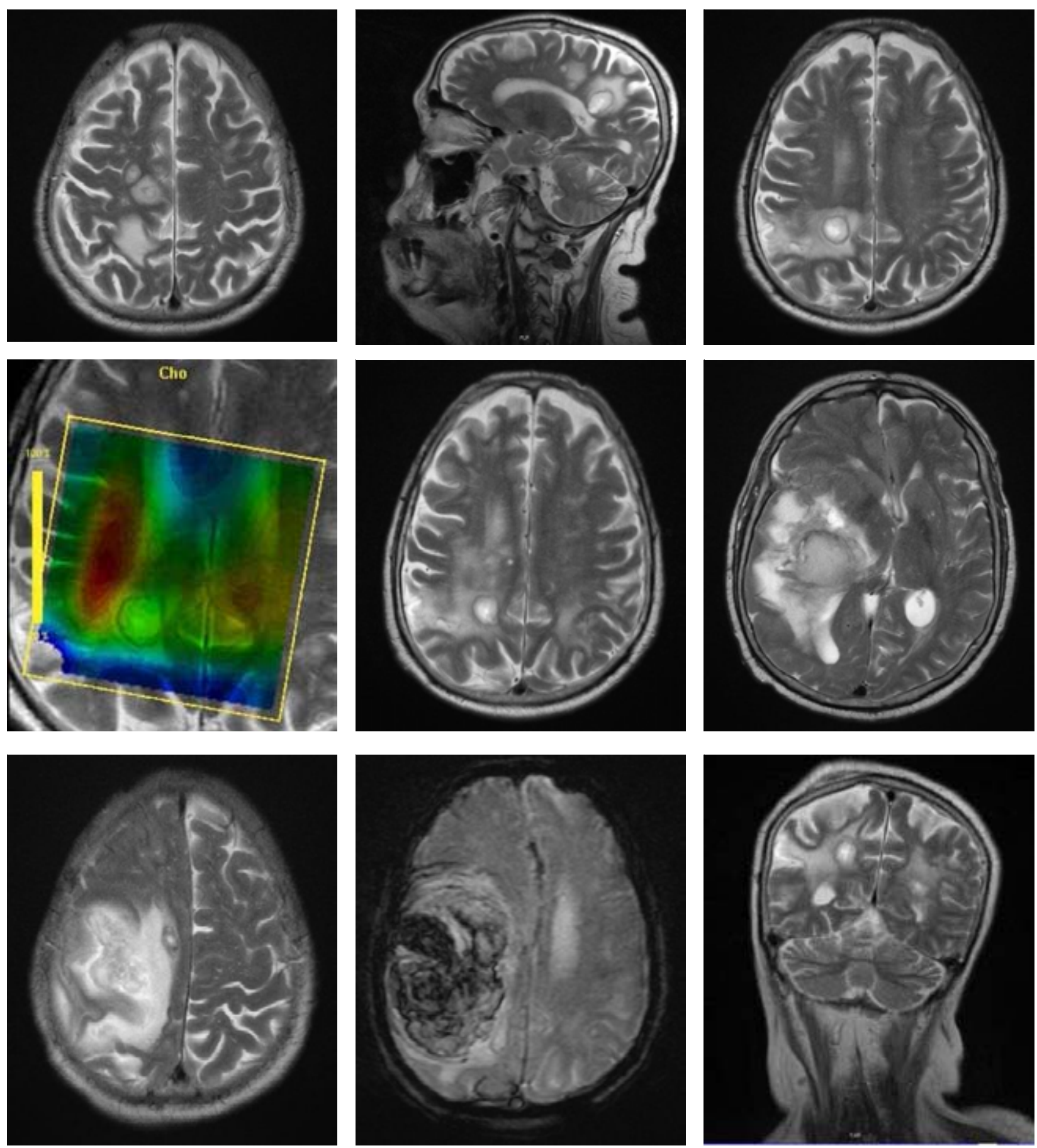

Figuras 1 - 9 Estudio imagenólogico. Fig.1, 2, 3. Múltiples lesiones secundarias a microangiopatía con edema perilesional y realce periférico anular a los 25 días post transplante Fig. 4, 5. Control escanográfico a los 32 días .

Fig. 6, 7. Control escanográfico a los 37 días. Fig. 8, 9. Control escanográfico a los 60 días.

quimatoso con sangre en el sistema ventricular y desviación leve de línea interhemisférica.

Se le realizo a drenaje neuroquirúrgico urgente del hematoma cerebral. El paciente fallece 2 días después (37 días postransplante cardiaco).

\section{DISCUSION}

El primer caso de Aspergilosis en seres humanos fue descrito en 1842 por Bennet en Edimburgo. Sin embargo los casos descritos correspondían a personas inmuno- 
competentes donde se presentaba como una enfermedad granulomatosa. No fue sino hasta 1950, cuando el uso de los esteroides y medicamentos citotóxicos se introdujo en la práctica diaria que se reconoció su papel como enfermedad oportunista y en 1953 se describe el primer caso de Aspergillosis Invasora (AI) en un paciente inmunocomprometido $(1,2)$.

En pacientes trasplantados de corazón la incidencia de esta infección parece ser menor que en pacientes trasplantados de otros órganos como hígado y pulmón. Sin embargo la mortalidad es igual de alta en estos pacientes y las dificultades existentes en cuanto al diagnóstico y manejo antimicótico son también similares $(1,3)$.

Se han identificado algunos factores de riesgo para desarrollar esta infección, tanto en pacientes transplantados de corazón como de otros órganos. Dentro de estos factores se destacan el uso de esteroides a altas dosis, el uso de antibióticos pretransplante, la colonización previa por Aspergillus, complicaciones quirúrgicas, el exceso de inmunosupresión, el rechazo crónico, la enfermedad por CMV, la ocurrencia en la institución de un episodio de Aspergillosis invasora en los dos meses previos o posteriores al trasplante y la necesidad de retrasplante $(2,4,5)$.

La especie aislada con mayor frecuencia es $\boldsymbol{A}$. fumigatus y la importancia de otras especies es menos dramática aunque se han diagnosticado infecciones por A. niger, A. flavus y A.terreus (3).

La incidencia de Aspergillosis invasiva en trasplantados cardiacos oscila entre 1 y 15\%, con una frecuencia en promedio de 5.2\%, representando el $69.8 \%$ de las infecciones micóticas, siendo la infección mas frecuente en su grupo $(4,6)$.

La Aspergillosis cerebral ocurre en el 10 a 40\% de las infecciones invasivas, sin embargo, es raro el compromiso aislado cerebral y es generalmente el resultado de diseminación desde otro órgano, generalmente los pulmones (2).

La mortalidad en trasplante cardiaco es del 53-78\% con aspergillosis invasora y cercana al 100\% si compromete el sistema nervioso central (4).

La infección se presenta con mayor frecuencia durante los primeros meses postransplante. El riesgo disminuye en forma importante después del primer año de transplante. Se ha observado una frecuencia de infección en los primeros 3 meses postransplante de 0.15 episodios/ paciente/mes y disminuye hasta 0.05 episodios/paciente/ mes despues de los tres meses $(5,7)$.

El SNC puede afectarse de distintas maneras en esta infección, incluyendo la formación de abscesos o granulomas en el parénquima cerebral, aracnoiditis difusa, infartos cerebrales o medulares, aneurismas micóticos o abscesos epidurales espinales $(4,7,8)$.

La aparición de abscesos cerebrales es un signo ominoso. Generalmente el paciente presenta síntomas focales. Cuando Aspergillus invade el sistema nervioso central por vía hematógena las hifas producen oclusión vascular o pueden atravesar las paredes capilares produciendo infarto hemorrágico que puede evolucionar a un infarto séptico con cerebritis y formación de abscesos. La distribución más común suele ser las regiones irrigadas por la arteria cerebral media y por la arteria cerebral anterior $(1,2,6)$.

Los síntomas son inespecíficos, y a pesar de su propensión a realizar invasión vascular los hemocultivos suelen ser negativos.

El diagnostico suele ser un reto y hasta el 18\% de los pacientes se diagnostican post mortem. La dificultad en el diagnóstico tiene varias razones:

1. En primer lugar la aspergillosis tiene presentaciones atípicas e inespecíficas que hacen primero pensar en otras infecciones que son además más frecuentes $(2,9)$.

2. Se presenta en un amplio y variado grupo de personas algunos de los cuales están expuestos por mucho tiempo al patógeno y otros que solo se exponen un tiempo corto $(3,8)$.

3. No hay un solo método diagnóstico. Se requieren varios exámenes y descartar otras infecciones antes de confirmar esta infección, cuyo patrón de oro para diagnosticarla es histopatológico y/o por cultivos y de cuya rapidez en el diagnóstico y tratamiento, dependerá la vida del paciente $(7,9)$.

El tratamiento de elección de la enfermedad invasora es el Voriconazol a una dosis de $8 \mathrm{mg} / \mathrm{kg} /$ día. Algunos expertos recomiendan como alternativa la administración de Anfotericina liposomal en dosis elevadas (10 mg/kg/día) basándose en estudios que demuestran una mayor eficacia sin mayor toxicidad. También es posible la utilización de Caspofungina (la única Equinocandina comercializada en nuestro país hasta el momento) que ha demostrado su utilidad en tratamientos de rescate $(8,9)$.

\section{REFERENCIAS}

1. Turgut, M.; Oncu, S. \& Tekin, C. (2008) Invasive fungal granuloma of the brain caused by Aspergillus fumigatus: a case report and review of the literature. Surg Neurol. 69:169-74

2. Singh, N. \& Pursell, K.J. (2008).Combination therapeutic approaches for the management of invasive aspergillosis in organ transplant recipients. Mycoses. 51:99-108

3. Haas, A. (2007). Invasive aspergillosis: epidemiology, diagnosis and management in immunocompromised patients. Drugs. 67:1567-601

4. Schwartz, S. \& Thiel, E. (2004). Update on the treatment of 
cerebral aspergillosis. Ann Hematol. 83 Suppl 1:S42

5. Muñoz, P. (2007). Infection in heart transplantation. Enferm Infecc Microbiol Clin. 25:587-97

6. Koffla, G. \& Otto, K. (2007). CNS aspergillosis: recognition, diagnosis and management. CNS Drugs. 21:659-76

7. Nadkarni, T. \& Goel, A. (2005). Aspergilloma of the brain: an overview. J Postgrad Med. 51 Suppl 1:S37-41
8. Silveira, M.P. (2007). Fungal infections in solid organ transplantation. Med. Mycol. 45:305-20

9. Drew, R.H. \& Davis, R. (2004).Comparative safety of amphotericin B lipid complex and amphotericin B deoxycholate as aerosolized antifungal prophylaxis in lung-transplant recipients. Transplantation. 77:232-7

10. Del Brutto, O.H. (2000) Infecciones Micóticas del Sistema Nervioso Central. Rev. Neurol. 30:447-459 